\section{Corneal hysteresis in patients with dry eye}

Turgut Ozal Medical Center Department of Ophthalmology, Inonu University, Malatya, Turkey

Correspondence: PG Firat, Inonu University, School of Medicine, Department of Ophthalmology, Firat Mahallesi, Hastane Caddesi, Malatya 44300, Turkey Tel: + 905063168038 ; Fax: + 9004223410660 . E-mail: penpe.firat@ inonu.edu.tr

Received: 18 February 2011 Accepted in revised form: 14 July 2011

Published online:

9 September 2011

\begin{abstract}
Purpose To measure corneal hysteresis and intraocular pressure (IOP) in patients with dry eye and to compare with normal subjects. Methods This cross-sectional study consists of 70 eyes of 40 patients with dry eye (group 1) and 75 eyes of 40 normal subjects (group 2). Eyes were diagnosed as dry eye or normal according to the clinical symptoms, biomicroscopical evaluation, and Schirmer test. Corneal hysteresis $(\mathrm{CH})$, corneal resistance factor (CRF), and corneacompensated intraocular pressure (IOPcc) were measured by the Ocular Response Analyser (ORA).

Results Mean $\mathrm{CH}$ values were $10.56 \pm 0.25 \mathrm{~mm} \mathrm{Hg}$ and $10.34 \pm 0.26 \mathrm{~mm} \mathrm{Hg}$, mean CRF values were $10.75 \pm 0.28 \mathrm{~mm} \mathrm{Hg}$ and $10.70 \pm 0.28 \mathrm{~mm} \mathrm{Hg}$, mean CCT values were $542 \pm 3.20 \mu \mathrm{m}$ and $543 \pm 3.89 \mu \mathrm{m}$ in group 1 and group 2, respectively. There was no statistically significant difference between the groups for these three parameters. IOPcc values measured with ORA were $15.73 \pm 0.36 \mathrm{~mm} \mathrm{Hg}$ in group 1 and $16.60 \pm 0.33 \mathrm{~mm} \mathrm{Hg}$ in group 2, and there was no statistically significant difference between the two groups.

Conclusions Corneal biomechanical parameters such as $\mathrm{CH}$ and CRF are not influenced in dry eye. Also statistical difference was not found between the two groups according to CCT and IOPcc values. Eye (2011) 25, 1570-1574; doi:10.1038/eye.2011.211; published online 9 September 2011
\end{abstract}

Keywords: dry eye; corneal hysteresis; intraocular pressure; ocular response analyser

\section{Introduction}

Dry eye is a disorder of the pre-corneal tear film layer. Deficiencies in secretion of tear or increased evaporation of tear are the main reasons of this clinical syndrome. It can be associated with different types of symptoms, signs, and ocular surface problems. Mainly it affects the cornea, conjunctiva, and the sclera. ${ }^{1}$ Although mild tear deficiency causes minimal ocular surface disease, a severe form of the disease can cause sight-threatening corneal complications. Corneal pathologies seen in dry eye are: epithelial keratopathy, filamentary keratitis, mucus plaque, marginal-paracentral corneal thinning, decrease in central corneal thickness, corneal ulceration, and perforation. ${ }^{2}$ There is a lot of evidence that inflammation is the main pathological factor but the exact mechanism has not been understood yet. All inflammatory cells, cytokines, and proapoptotic factors were found to be increased in dry eye. ${ }^{3,4}$ In an experimental study, corneal peripheral and central epithelium apoptosis were shown in dry eye. ${ }^{5}$ Elsheikh et $a l^{6}$ showed that an intact corneal epithelium has a very important function over the corneal biomechanics. But the effect of pre-corneal tear film layer, which was accepted as a member of corneal epithelium, over the corneal biomechanics has not been clarified yet.

IOP measurement in dry eye is another pending question. The accuracy of GAT depends on some simplifying assumptions and one of them is that the surface tension of the tear film would balance the corneal rigidity. As dryeye patients lack a healthy tear film layer, IOP measurement with GAT can be erroneous. ${ }^{7}$ Another factor is central corneal thickness (CCT), which is shown to be decreased in dry eye. ${ }^{8,9}$ The consensus is that IOP measurement using GAT may be falsely reduced in patients with thin corneas. ${ }^{7}$

All these corneal changes seen in dry eye can affect the corneal viscoelasticity, which can be measured with the Ocular Response Analyser (ORA; Reichert Ophthalmic Instruments, Buffalo, NY, USA). Corneal hysteresis $(\mathrm{CH})$ is the term that is used for corneal viscoelastic properties. The ORA 
determines corneal biomechanical properties using an applied force-displacement relationship. The cornea passes a first applanation and then comes a slight concave form. Within a few milliseconds, applied pressure decreases and the cornea passes through an applanated state again. It returns from concave form to its original convex curvature. All these processes take place within approximately 20 milliseconds. The measurements are taken from a $3.00-\mathrm{mm}$ diameter central corneal area. During this applanation process, two different applanation pressures are noted because of corneal viscoelastic properties. ${ }^{10} \mathrm{So}$, we can derive corneal viscoelastic properties such as $\mathrm{CH}$, corneal resistance factor (CRF), and corneal-compensated intraocular pressure (IOPcc) in numerical value. Although $\mathrm{CH}$, also known as corneal damping response, reflects corneal viscoelasticity, CRF reflects corneal elasticity. ${ }^{11,12}$

In this study we used the ORA to measure the corneal biomechanical properties and IOP. The aim of this study was to investigate whether the pre-corneal tear film layer deficiency has an effect on corneal biomechanics measured with ORA.

\section{Materials and methods}

\section{Subjects}

This study consisted of 70 eyes of 40 dry-eye patients (group 1) and 75 eyes of 40 normal subjects (group 2). We certify that all applicable institutional and governmental regulations concerning the ethical use of human volunteers were followed during this research. All patients completed the informed consent form. The diagnosis of dry eye is difficult because it does not have a standard test or specific findings on biomicroscopy. The diagnosis is usually made according to the combined symptoms, clinical examination, and specific tests. Eyes were diagnosed as dry eye or normal according to the clinical symptoms, biomicroscopic evaluation, and Schirmer I test. A questionnaire form was also used for diagnosis (Table 1).

Subjects underwent a full ophthalmological examination, including best-corrected visual acuity, IOP measuring by applanation tonometry, biomicroscopic,

Table 1 Questionnaire form for dry eye

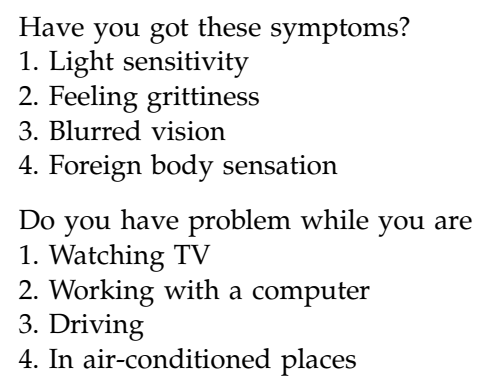

and fundus examination by slit lamp. Further clinical tests were performed on patients that had two or more symptoms according to the questionnaire form. Schirmer I test was performed on all patients. A combination of positive symptoms according to the form and Schirmer I test values less than $10 \mathrm{~mm}$ was accepted as dry eye. Patients having refraction error, keratoconus, previous intra or extra ocular surgery history, blepharitis, conjunctivitis, corneal dystrophy, cataract, glaucoma, and patients using any medications were excluded from the study.

\section{Ocular response analyser measurement}

$\mathrm{CH}, \mathrm{CRF}$, and IOPcc were measured by the ORA. The interval between the ORA and GAT measurements was $30 \mathrm{~min}$. Three GAT measurements were taken first and then ORA measurements were taken by an observer masked in terms of groups. Three ORA measurements were obtained per eye. The measurement with the highest waveform score was retained.

Central corneal thickness (CCT) of the patients was measured after application of a local anaesthetic agent with an ultrasonic pachymeter attached to the ORA.

\section{Statistical analyses}

Statistical analyses were performed using SPSS v.16. (SPSS Inc., Chicago, IL, USA). Results are presented as mean $\pm S D$. Student's $t$-test was used to assess the difference in parameters between the groups. Pearson correlation test was used to assess the correlation between $\mathrm{CH}$ and IOPcc. A $P$-value less than 0.05 was accepted as being statistically significant.

\section{Results}

The study included 70 eyes of 40 subjects in the dry-eye group and 75 eyes of 40 subjects in the normal group. Patients with dry eye consisted of 24 women (60\%) and 16 men (40\%). In the normal group, 22 women (55\%) and 18 men $(45 \%)$ were included. Mean age for group 1 was $45.45 \pm 7.97$ years, and $43.23 \pm 8.50$ years for group 2 . There was no statistical difference between groups for age and sex $(P \geq 0.05)$ (Table 2$)$.

Table 2 Demographic characteristics of patients

\begin{tabular}{lccc}
\hline & Group 1 & Group 2 & $P$ \\
\hline Female/male & $24 / 16$ & $22 / 18$ & $P \geq 0.05$ \\
Age & $45.45 \pm 7.97$ & $43.23 \pm 8.50$ & $P \geq 0.05$ \\
OD/OS & $37 / 33$ & $39 / 36$ & $P \geq 0.05$ \\
\hline
\end{tabular}

Group 1: dry eye patients.

Group 2: normal subjects. 
The mean $\mathrm{CH}$ was $10.56 \pm 0.25 \mathrm{~mm} \mathrm{Hg}$ and CRF was $10.75 \pm 0.28 \mathrm{~mm} \mathrm{Hg}$ in group 1, and $10.34 \pm 0.26 \mathrm{~mm} \mathrm{Hg}$ and $10.70 \pm 0.26 \mathrm{~mm} \mathrm{Hg}$ in group 2, respectively $(P>0.05)$. The mean CCT was $542.78 \pm 3.29 \mu \mathrm{m}$ in group 1 and $543.91 \pm 3.89 \mu \mathrm{m}$ in group $2(P>0.05)$ (Figure 1).

IOPcc is another parameter, which was measured with ORA. The mean IOPcc in group 1 was $15.73 \pm 0.36 \mathrm{~mm} \mathrm{Hg}$ and $16.60 \pm 0.33 \mathrm{~mm} \mathrm{Hg}$ for group $2(P \geq 0.05)$ (Figure 2).

There was no difference between the groups according to the signal pattern. The signal produced by the infrared light in a normal eye and a dry eye is given in Figure 3.

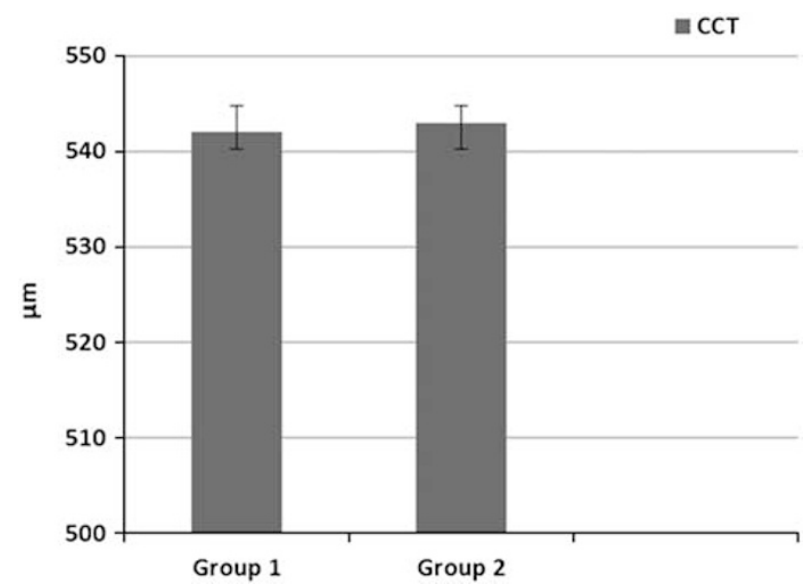

Figure 1 Comparison of CCT values between groups. The error bars indicate the standard deviation of the values (group 1: dryeye patients; group 2: normal subjects; CCT, central corneal thickness).

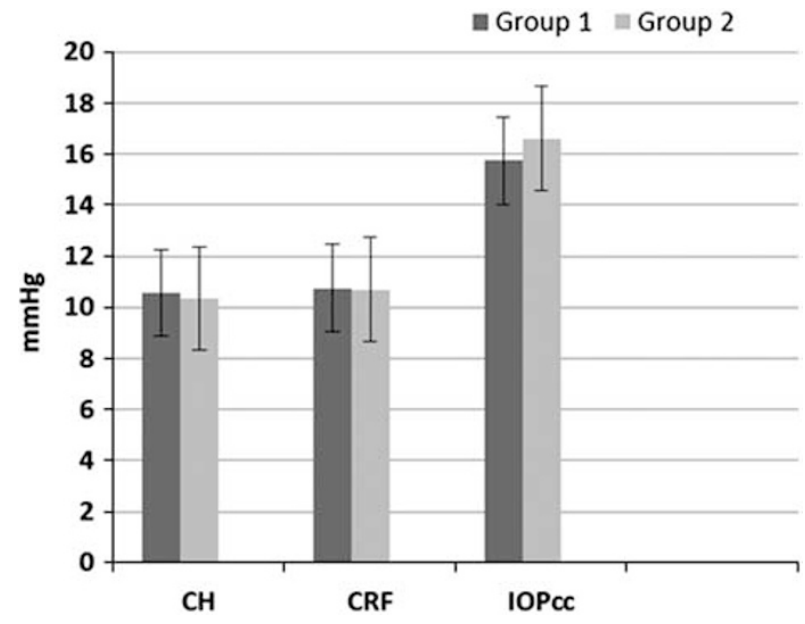

Figure 2 Comparison of $\mathrm{CH}, \mathrm{CRF}$ and IOPcC between groups. The error bars indicate the standard deviation of the values (group 1: dry-eye patients; group 2: normal subjects; $\mathrm{CH}$, corneal hysteresis; CRF, corneal resistance factor; IOPcc, corneal-compensated intraocular pressure).

\section{Discussion}

The cornea is the most affected tissue in dry eye, so the changes in the cornea are very important in diagnosis, treatment, and management of the dry eye, and also diseases related with dry eye. Glaucoma is the bestknown disease related with dry eye. Not only glaucoma but also medications for glaucoma cause dry eye. ${ }^{13}$ As IOP is related to the corneal biomechanical properties 'are we measuring the IOP accurately with dry eye' is the question we must answer to make a true diagnosis and management with glaucoma patients. In this study, we aimed to measure the corneal biomechanical properties by ORA. Consecutive corneal measurements can be taken with ORA. ${ }^{14}$ We can estimate that IOP by ORA is less influenced by corneal properties. ${ }^{10}$ In this way, we can say that ORA is a device that measures corneal properties and IOP together.

Our study includes 70 eyes of 40 dry-eye patients and 75 eyes of 40 normal subjects. According to Laiquzzaman et $a l^{15} \mathrm{CH}$ of normal subjects have the same values in both eyes, so right and left eyes were taken in one group. Diurnal variation of $\mathrm{CH}$ was evaluated and it was seen that $\mathrm{CH}$ values were steady within $24 \mathrm{~h} .{ }^{15,16}$ The mean $\mathrm{CH}$ value was calculated by three consecutive measurements taken in $5 \mathrm{~min}$. $\mathrm{CH}$ was $10.56 \pm 0.25 \mathrm{~mm} \mathrm{Hg}$ in the dry-eye group and $10.34 \pm 0.26 \mathrm{~mm} \mathrm{Hg}$ in the normal population. There was no statistically significant difference between the two groups $(P \geq 0.05)$. Mean CRF was $10.75 \pm 0.28 \mathrm{~mm} \mathrm{Hg}$ in the dry-eye group and $10.70 \pm 0.26 \mathrm{~mm} \mathrm{Hg}$ in the normal group, respectively. There was no statistically significant difference between the two groups too $(P \geq 0.05)$. Although dry eye affects the cornea and causes serious problems, it does not affect the biomechanical properties of the cornea. Luce ${ }^{11}$ reported the

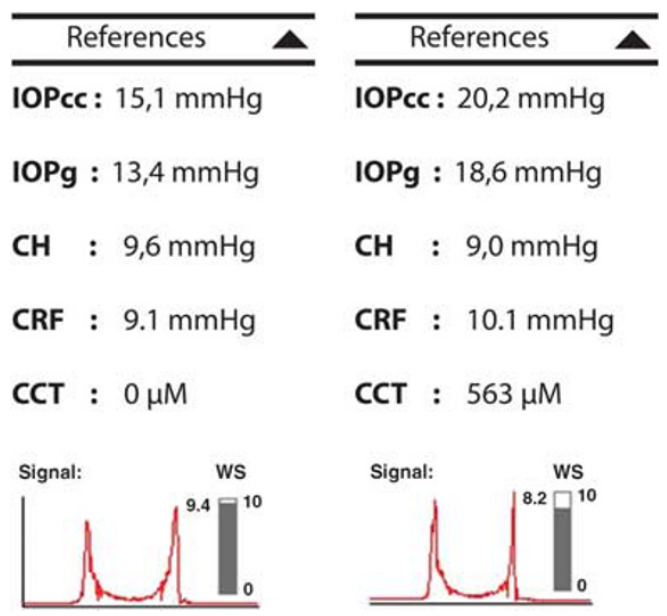

Figure 3 Ocular response analyser measurements of the patients. The figure on the right belongs to a normal subjects and the figure on the left belongs to a dry-eye patient. 
decreased $\mathrm{CH}$ measurements in eyes having LASIK and Fuchs dystrophy. In this study, $\mathrm{CH}$ and $\mathrm{CRF}$ values were a little higher than the normal subjects. There was no statistical difference between the groups, so we can say that in contrast to these diseases, corneal biomechanical properties are kept intact in dry-eye syndrome.

Mean CCT was $542.78 \mu \mathrm{m}$ in the dry-eye group and $543.91 \mu \mathrm{m}$ in the normal group. Although CCT in the dry-eye group is a little lower than the normal group, there was no statistically significant difference between the two groups within this parameter $(P \geq 0.05)$. Previous studies showed a decrease in CCT in dry-eye syndrome, because of the osmolality of tear film and hypertonic tear film. ${ }^{8,9}$ According to our results, a decrease in CCT is not related with corneal biomechanical changes in dry eye. In another study, an increase in CCT values was seen at night but corneal viscoelasticity did not change. ${ }^{15}$ This is explained because of the change in hydration state, which can be said for dry-eye syndrome too. Karadayı et $a l^{17}$ showed that the CCT in dry eyes increases rapidly after application of artificial tears. They suggest this finding as a diagnostic criterion for dry eye.

IOP is another important parameter. IOP measured by ORA is called cornea-compensated IOP because it does not get influenced by corneal parameters. Mean corneal-compensated IOP (IOPcc) was $15.73 \pm 0.36 \mathrm{~mm} \mathrm{Hg}$ in the dry-eye and $16.60 \pm 0.33 \mathrm{~mm} \mathrm{Hg}$ in the normal group. There was no statistical difference between these two groups $(P \geq 0.05)$, so this proves to us that ORA is not influenced by CCT. In several studies that were made with Goldman applanation tonometry (GAT), there was a relationship between CCT and IOP values. GAT measured IOP accurately when CCT was $520 \mu \mathrm{m}$, and each $10 \%$ difference of CCT would result in $1.1 \mathrm{~mm} \mathrm{Hg}$ differences in IOP. ${ }^{18-20}$ In this study, the correlation between $\mathrm{CH}$ and IOPcc was also searched. There was a positive correlation and this is statistically significantly different (Pearson correlation test, $r=0.466, P \leq 0,05)$.

Also, examination of the ORA signal morphology provides important reports about the corneal biomechanical properties. The ORA applies a focused stream of air to the external surface of the cornea. In a recent study done by Liu and Roberts, ${ }^{21}$ deflection at applanation of the cornea is the subtraction of the internal pressure from the external air pressure applied to the cornea and the surface tension of the tear film. The literature states that the tear film pressure is $4.15 \mathrm{~mm} \mathrm{Hg} .{ }^{7}$ During the ORA measurement, the cornea passes a first applanation and then comes a slight concave form. In this concave state, tear film tension pulls the cornea back toward applanation. ${ }^{22}$ The reflection of the infrared light gives indirect evidence that the tear film remains intact in healthy eyes. In this current study we performed ORA measurements on dry-eye patients, but we did not see any difference in the reflection of the infrared light between dry eye and normal subjects. The same signal morphology of the dry eye and normal subjects leads to the conclusion that deficiency of the tear film does not alter the biomechanical properties of the cornea.

Our study had some limitations. The number of the patients with dry eye was small and dry-eye patients did not group according to the severity of the disease in subgroups. To our knowledge, our study is the first investigating the corneal biomechanical properties with ORA in dry eye syndrome.

In conclusion, dry-eye syndrome affects the cornea and causes severe symptoms but does not cause biomechanical changes on the cornea. CCT decrease is a result of osmolality of tear film. Further studies must be done with larger groups.

\section{Summary}

\section{What was known before}

- Dry eye is a disorder of pre-corneal tear film layer. Although severe forms can cause sight-threatening corneal complications, corneal biomechanical changes are not known in dry eye.

What this study adds

- Dry-eye syndrome affects cornea and causes severe symptoms but it does not cause biomechanical changes on the cornea.

\section{Conflict of interest}

The authors declare no conflict of interest.

\section{References}

1 Stern ME, Beuerman WE, Fox RI, Gao J, Mircheff AK, Pflugfelder SC. The pathology of dry eye: the interaction between the ocular surface and lacrimal glands. Cornea 1998; 17: 584-589.

2 Farris RL. Abnormalities of the tears and treatment of dry eyes. In: Kaufman HE, Barron BA, Mcdonald MB (eds). The Cornea. Butterworth-Heinemann: Newton, 1998, pp 109-129.

3 Pflugfelder SC, Jones D, Ji Z, Afonso A, Monroy D. Altered cytokine balance in the tear fluid and conjunctiva of patients with Sjögren's syndrome keratoconjunctivitis sicca. Curr Eye Res 1999; 19: 201-211.

4 Brignole F, Pisella PJ, Goldschild M, De Saint Jean M, Goguel A, Baudouin C. Flow cytometric analysis of inflammatory markers in conjunctival epithelial cells of patients with dry eyes. Invest Ophthalmol Vis Sci 2000; 41: 1356-1363. 
5 Yeh S, Song XJ, Farley W, Li DQ, Stern ME, Pflugfelder SC. Apoptosis of ocular surface cells in experimentally induced dry eye. Invest Ophthalmol Vis Sci 2003; 44: 124-129.

6 Elsheikh A, Alhasso D, Rama P. Assessment of the epithelium's contribution to corneal biomechanics. Exp Eye Res 2008; 86: 445-451.

7 Damji KF, Muni RH, Munger RM. Influence of corneal variables on accuracy of intraocular pressure measurement. J Glaucoma 2003; 12: 69-80.

8 Dayanır V, Sakarya V, Ozcura F, Kir E, Aktunç T, Ozkan BS et al. Effect of corneal drying on central corneal thickness. J Glaucoma 2004; 13: 6-8.

9 Cohen EM, Polse KA, Brand RJ, Mandell RB. Humanity effects on corneal hydration. Invest Ophthalmol Vis Sci 1990; 31: 1282-1287.

10 Medeiros FA, Weinreb RN. Evaluation of the influence of corneal biomechanical properties on intraocular pressure measurements using the ocular response analyzer. J Glaucoma 2006; 15: 364-370.

11 Luce DA. Determining in vivo biomechanical properties of the cornea with an ocular response analyzer. J Cataract Refract Surg 2005; 31: 156-162.

12 Broman AT, Congdon NG, Bandeen-Roche K, Quigley HA. Influence of corneal structure, corneal responsiveness, and other ocular parameters on tonometric measurement of intraocular pressure. J Glaucoma 2007; 16: 581-588.

13 Leung EW, Medeiros FA, Weinreb RN. Prevalence of ocular surface disease in glaucoma patients. J Glaucoma 2008; 17: 350-355.

14 Moreno-Montanes J, Maldonado MJ, Garcia N, Mendiluce L, García-Gómez PJ, Seguí-Gómez M. Reproducibility and clinical relevance of the Ocular response Analyzer in nonoperated eyes: corneal biomechanical and tonometric implications. Invest Ophthalmol Vis Sci 2008; 49: 968-974.

15 Laiquzzaman M, Bhojwani R, Cunlife I, Shah S. Diurnal variation of ocular hysteresis in normal subjects: relevance in clinical context. Clin Exp Ophthalmol 2006; 34: 114-118.

16 Kida T, Liu JHK, Weinreb RN. Effect of $24 \mathrm{~h}$ corneal biomechanical changes on intraocular pressure measurement. Invest Ophthalmol Vis Sci 2006; 47: 4422-4426.

17 Karadayı K, Ciftci F, Akin T, Bilge AH. Increase in central corneal thickness in dry and normal eyes with application of artificial tears: a new diagnostic and follow-up criterion for dry eye. Ophthalmol Physiol Opt 2005; 25: 485-491.

18 Ehlers N, Bramsen T, Sperling S. Applanation tonometry and central corneal thickness. Acta Ophthalmol 1975; 53: 34-43.

19 Mendelsohn AD, Forster RK, Mendelsohn SL. Comparative tonometric measurements of eye bank eyes. Cornea 1987; 6: 219-225.

20 Doughty MJ, Zaman ML. Human corneal thickness and its impact on intraocular pressure measures: a review and meta-analysis approach. Surv Ophthalmol. 2000; 44: 367-408.

21 Liu J, Roberts CJ. Influence of corneal biomechanical properties on intraocular pressure measurement. J Cataract Refract Surg 2005; 31: 146-155.

22 Glass DH, Roberts CJ, Litsky AS, Weber PA. A viscoelastic biomechanical model of the cornea describing the effect of viscosity and elasticity on hysteresis. Invest Ophthalmol Vis Sci 2008; 49: 3919-3926. 